\title{
Your Activity Tracker Knows When You Quit Smoking
}

\author{
Ken Kawamoto \\ Hitachi, Ltd. \\ Central Research Lab \\ 1-280 Higashi Koigakubo, \\ Kokubunji, Tokyo, Japan \\ ken.kawamoto.qg@hitachi.com \\ Takeshi Tanaka \\ Hitachi, Ltd. \\ Central Research Lab \\ 1-280 Higashi Koigakubo, \\ Kokubunji, Tokyo, Japan \\ takeshi.tanaka.nz@hitachi.com
}

\author{
Hiroyuki Kuriyama \\ Hitachi Solutions, Ltd. \\ 4-12-7 Higachi Shinagawa, \\ Shinagawa, Tokyo, Japan \\ hiroyuki.kuriyama.cd@hitachi- \\ systems.com
}

\begin{abstract}
This paper discusses outcomes of our exploratory research aiming to discover ways of utilising continuous long term respiratory rate data collected from actigraphy (wrist-worn accelerometers.) We show that by monitoring changes in respiratory rate during sleep, we can detect and visualise various physical conditions that were previously not detectable using such simple wearable sensors, namely; the subjective level of drunkenness, fever, and smoking cessation. This study provides valuable insight into the potential of actigraphy, not simply as a tool for detecting common daily activities, but as a base for building a generic lifelog system that can evaluate the more qualitative aspects of your life.
\end{abstract}

\section{Author Keywords}

Actigraphy; activity tracker; respiratory rate; drunkenness; fever; smoking detection

\section{ACM Classification Keywords}

H.5.m. Information interfaces and presentation (e.g., HCI): Miscellaneous

\section{INTRODUCTION}

Actigraphs (wristband based accelerometers), also popularly known as activity trackers, are one of the most commercially successful types of wearable sensors that have made its way into the lives of millions of consumers worldwide [6-8]. Some of the advantages that actigraphs offer over their counterparts (e.g: cameras, positioning systems, heart rate monitors) are that they are cheaper, less intrusive, and can detect a wide range of daily activities. Such attributes make actigraphs an attractive tool to base a lifelog system on.

Permission to make digital or hard copies of all or part of this work for personal or classroom use is granted without fee provided that copies are not made or distributed for profit or commercial advantage and that copies bear this notice and the full citation on the first page. Copyrights for components of this work owned by others than ACM must be honored. Abstracting with credit is permitted. To copy otherwise, or republish, to post on servers or to redistribute to lists, requires prior specific permission and/or a fee. Request permissions from Permissions@acm.org.

ISWC'14, September 13 - 17, 2014, Seattle, WA, USA

Copyright 2014 ACM 978-1-4503-2969-9/14/09...\$15.00.

http://dx.doi.org/10.1145/2634317.2634327
Our interest is in building an actigraph-based lifelog system that can fully automate the generation of currently handwritten diaries. Such systems may be useful simply as a tool for personal reflection, but we also see its potential for "activity mining", the use of data mining on activity logs to understand how we can live a more abundant life [1].

The majority of the past research in actigraphy have focused on recognising common daily activities (eg: sleep, household chores, and various forms of exercises[2,3]). However, being able to log "what" you did solves only part of the requirements of diaries; a proportion of the contents of diaries is dedicated to recording our physical and mental conditions (i.e "how" we felt physically, and emotionally).

In the past, we have approached the problem of physical condition monitoring from various angles including fitness evaluation and sleep quality evaluation [4]. As an unexpected side-effect of the latter research, we discovered that examination of actigraphic data during sleep allows accurate estimation of the respiratory rate (the rate of breathing)[4]. Respiratory rates are currently usually measured using nasal airflow sensors or chest wall sensors, which are both expensive and invasive [5]. The significance of our findings in [4] is that it allows long term continuous measurements of respiratory rates, which was particularly difficult with prior art. Due to this, as far as the authors are aware, very little research has been done in the field of continuous long term respiratory rate recordings.

The purpose of this study is to explore how we can utilise long term respiratory rate data for physical condition evaluation. In this paper, we present major findings from this study, namely that respiratory rates measured from actigraphs enable the identification of conditions like inebriation (drunkenness), fever, and smoking cessation. We will not be discussing the biological mechanisms behind these findings; we will merely present the statistical facts observed in our study.

\section{DATA COLLECTION}

For this study, we used actigraphic data collected continuously over the course of 6 years (2007 2014) from 20 individuals. The device employed was a "Life Microscope" wristband (Hitachi Ltd.)[1] that measures triaxial acceleration at a resolution of $11.7 \mathrm{mG}$ at $20 \mathrm{~Hz}$. All individuals wore the wristband all day and night, other than when charging the batteries (every fortnight or so). 
We processed the actigraphic data according to the method detailed in [4]. To explain the idea briefly; acceleration data collected from the wrist during sleep contain tiny movements that have propagated from the chest wall's breathing movements. By performing frequency analysis, we can extract an estimate of the respiratory rate from this movement. The results are sparse (detection rate: $44 \%$ ), since the propagation of the chest's movements depend on the posture, but the method provides an accurate (mean absolute error: 0.52 counts $/ \mathrm{min}$ ) estimation of respiratory rate during sleep, simply by wearing a wristband.

Some of the individuals kept subjective, handwritten records of their daily activities. The records of two of the individuals included subjective reports of their inebriation (drunkenness) before going to sleep on a VAS scale from 0 (no alcohol taken) to 5 (heavily inebriated). Two individuals reported days when they had fever. Two individuals reported to have quit smoking during the recorded period. Note that none of these self reports were originally intended for data analysis, so no precautions were made beforehand to ensure that the subjective reports maintained consistency between individuals, over time, or against some global criterion.

\section{INEBRIATION DETECTION}

On inspecting the collected recordings, we witnessed occasional nights where the respiratory rate surged in the first hour or so, then fell back to normal afterwards. Fig.1 shows four examples of this, where the surges are labelled with arrows. Upon cross examination of the data with the handwritten records, we noticed that they matched nights where the individuals had reported a non-zero inebriation level. This is not entirely unlikely, since it is known that alcohol act as stimulants, causing temporary increase of heart rate. We thus explored the possibility of predicting nights on which individuals felt inebriated.

\section{Data}

The data employed were from two individuals that recorded nightly levels of inebriation, for a total of 753 days. The individuals marked a score of 0 on days when they took no alcohol, a score of 5 on days when they felt heavily inebriated, and in-between depending on how they felt.

To visualise the relation of subjective inebriation with the respiratory rate, we evaluated the "surge amount" for each night, defined as follows. We calculated the mean

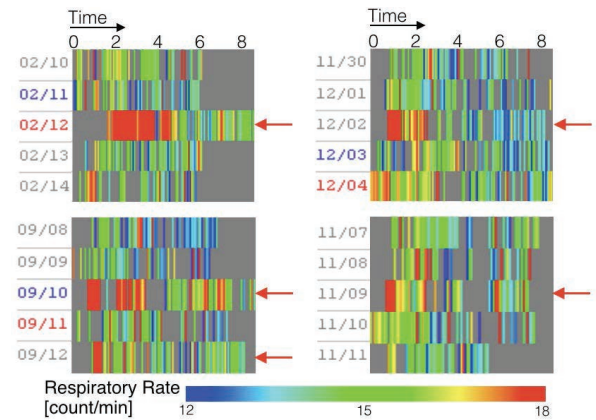

Figure 1. Nights with surges at sleep onset (shown with red arrows)
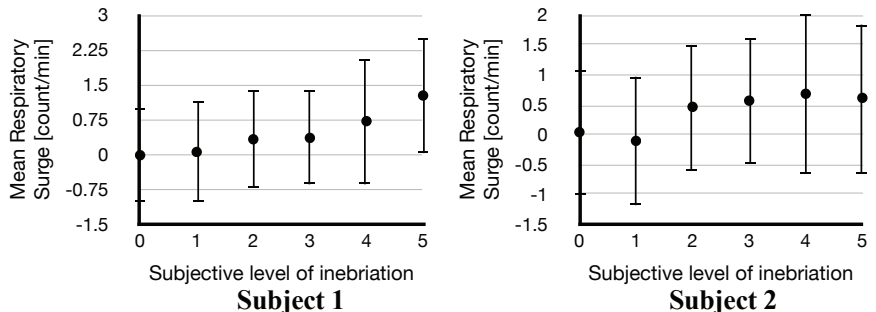

Figure 2. Mean respiratory rate surge amount per inebriation level (error bar= standard deviation)

respiratory rate $\operatorname{avg}_{0,90}[t]$ for the first 90 minutes of sleep for each night $t$, and then took the increase in $\operatorname{avg}_{0,90}[t]$ compared with the past 7 days:

$$
\operatorname{diff}_{0,90}[t]=\left(\operatorname{avg} g_{0,90}[t]\right)-\left(\sum_{i=1}^{7} a v g_{0,90}[t-i]\right)
$$

Fig.2 shows the mean of diff $0,90[t]$ for each recorded level of intoxication for the two individuals. For both individuals, the mean surge amount rises as the level of inebriation rises. There is a significant $(\mathrm{p}<0.05)$ difference between days recorded 0 and days recorded above 2 .

\section{Predictive power of respiratory rates for inebriation}

We now attempt to separate "drunk" days with recorded inebriation of above 2 (chosen arbitrarily), from "sober" days with 0 ("no alcohol taken"), by performing binary classification on surge level features. For each night, we calculate surge features with a sliding window (diff $0,90[t]$, $\operatorname{diff}_{90,180}[t]$, diff $\left.180,270[t]\right)$, and train an SVM classifier for two target classes: "sober" and "drunk". We evaluate the effectiveness of this method by leave-one-out cross validation for each individual separately, and both subjects together.

\section{Results}

Table 1 shows the results of the evaluation. The positive predictive ratio (precision of "Drunk") is high (73\%) for subject 1 , and fairly low (36\%) for subject 2 . Note that the ratio of "Drunk" days in the original data is also very low

\begin{tabular}{|l|l|r|r|r|r|r|}
\hline \multicolumn{2}{|l|}{ Subject 1 } & \multicolumn{2}{|l|}{ Predicted } & \multicolumn{3}{l|}{} \\
\cline { 3 - 7 } \multicolumn{2}{|l|}{} & Drunk & Sober & Ratio & Prec. & Recall \\
\hline \multirow{2}{*}{ Actual } & Drunk & 93 & 70 & $54.7 \%$ & 0.73 & 0.57 \\
\cline { 2 - 8 } & Sober & 35 & 100 & $45.3 \%$ & 0.59 & 0.74 \\
\hline
\end{tabular}

\begin{tabular}{|l|l|r|r|c|r|r|}
\hline \multicolumn{2}{|l|}{ Subject 2 } & \multicolumn{2}{|l|}{ Predicted } & \multicolumn{3}{l|}{} \\
\cline { 2 - 7 } \multicolumn{2}{|l|}{} & Drunk & Sober & Ratio & Prec. & Recall \\
\hline \multirow{2}{*}{ Actual } & Drunk & 40 & 31 & $24.0 \%$ & 0.36 & 0.56 \\
\cline { 2 - 8 } & Sober & 72 & 147 & $76.0 \%$ & 0.83 & 0.67 \\
\hline
\end{tabular}

\begin{tabular}{|l|l|r|r|r|r|r|}
\hline \multicolumn{2}{|l|}{ Both } & \multicolumn{2}{|l|}{ Predicted } & \multicolumn{3}{l|}{} \\
\cline { 2 - 7 } \multicolumn{2}{|l|}{} & Drunk & Sober & Ratio & Prec. & Recall \\
\hline \multirow{2}{*}{ Actual } & Drunk & 138 & 96 & $39.8 \%$ & 0.57 & 0.59 \\
\cline { 2 - 7 } & Sober & 104 & 250 & $60.2 \%$ & 0.72 & 0.71 \\
\hline
\end{tabular}

Table 1. Results of "Drunk" and "Sober" classification for individual subjects, and both subjects 
for subject 2, which explains the low precision. The precision in all cases are significantly higher than chance level. When evaluating using data from both individuals combined, the positive predictive value is $57 \%$, and the balanced accuracy (accuracy accounting for imbalance in the training set) is $65 \%$.

\section{Discussion}

We showed that respiratory rate data exhibits surges at sleep onset on days with subjective inebriation, and that we are able to predict days in which individuals subjectively felt "drunk" by using only data from an actigraph. There were some limitations to the setup which, when solved, may improve the prediction accuracy. The training data was based on a subjective self-report that was not normalised nor guaranteed to be consistent. There were also no records of the quantity of alcohol intake, or time of the last intake. A better experiment setup in the future would be to record the alcohol intake time and amount, and to take objective inebriation level measurements (e.g blood/breath alcohol content, or coordination tests.)

While our current setup and results only lets us conclude that actigraphs can predict days with subjective inebriation, future work with more objective data may let us predict the objective inebriation level. To be able to do this just by wearing a wristband will have significance for day-to-day healthcare, as well as specific situations like prevention and treatment of alcohol abuse.

\section{FEVER DETECTION}

We found that with many individuals, on some days every year or so, there are consecutive days where the respiratory rate is high for the entire night. Fig.3 shows several examples of this; it clearly shows the red region (high respiratory rate) extending across several nights. Examination of handwritten records revealed that they matched days when the individuals had "fever". This in itself is understandable, as the core body temperature

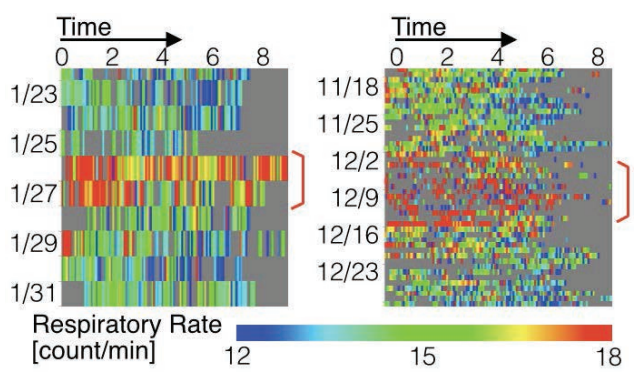

Figure 3. Examples of respiratory surges during fever

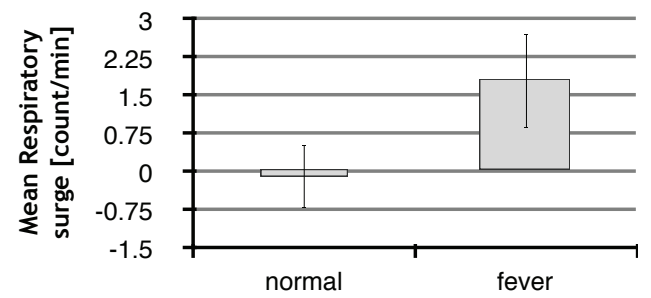

Figure 4. Respiratory rate surge for normal days, and days with fever relates closely to the respiratory rate. From this observation, we considered the possibility of detecting fever from respiratory rate data.

\section{Data}

There were two individuals that recorded days when they had fever. In a span of 800 days, there were 4 separate regions where the individuals reported a fever (high temperature), for a total of 17 days. The level of fever at which they reported is thought to differ individually and is not known, nor are the actual body temperatures at those times.

Again, to visualise the relation between fever and rise in respiratory rate, we calculate $\operatorname{diff} f_{0,360}[t]$, i.e the increase in mean respiratory rate from sleep onset to 6 hours into sleep, compared to the past 7 days. As shown in Fig.4, days with fever have significantly higher surge level $(p=4.27 \mathrm{E}-06)$ compared to normal days.

Due to the lack in the number of training cases of fever, we made no attempts to evaluate the predictive power of respiratory rate for finding days with fever. However, we found that simply labelling days with surge rate above 1.5 as "fever" and others as "normal" allowed classification with balanced accuracy of $70 \%$. Note that this figure should only be regarded as a very preliminary result, since the training case (which were used to derive the threshold of 1.5) and the test case is the same.

\section{SMOKING CESSATION DETECTION}

Finally, we demonstrate that respiratory rate data can be used to determine if and when individuals quit smoking. Fig. 5 shows an example of an individual who quit smoking on September 2, 2010. Fig.5 (b) is a plot of the mean respiratory rate, normalised for seasonal differences; the mean respiratory rate at each day of the year is calculated for the individual, and then is deducted from the mean respiratory rate of that date. The respiratory rate falls by 1.66 counts/min from the day after cessation. Another individual who also quit smoking displayed similar tendencies.

\section{Prediction of the cessation date}

There were only two individuals who quit smoking in our dataset. One, shown in Fig. 5, went from smoking an

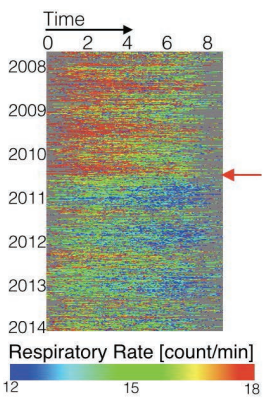

(a)

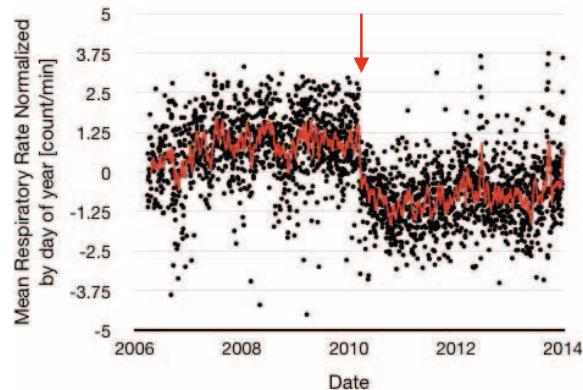

(b)
Figure 5. Long term respiratory rate data of an individual who quit smoking (a) as a heat map (b) as a plot of the mean nightly respiratory rate, normalised by day of year (day of cessation shown with arrows) 

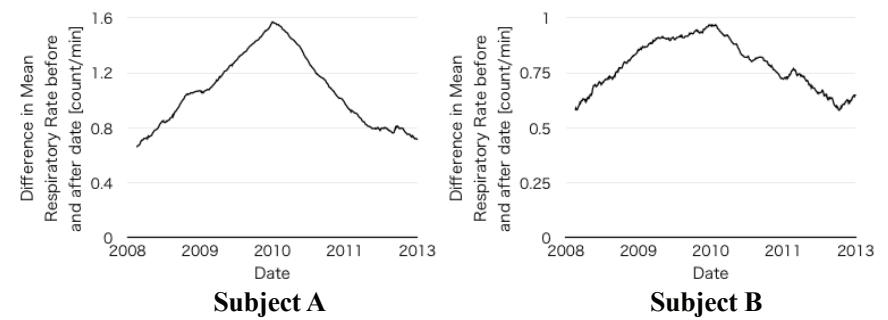

Figure 6. Difference in respiratory rate before and after each date.

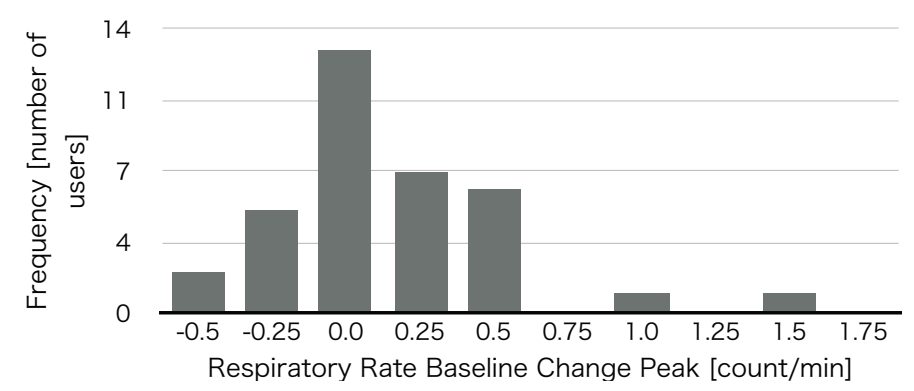

Figure 7. Histogram of the peak respiratory rate baseline change level for each individual. The only two individuals who quit smoking stand out outside $1 \sigma$

average of 15 cigarettes a day to none. The other went from smoking an average of 10 cigarettes a day, to a couple a month.

\section{Cessation date prediction}

For these two individuals, we attempt to predict the day on which they quit smoking, by finding the day the largest shift in respiratory rate baseline occurs. We thus find the largest peak in the change of respiratory rate; for any particular day $t$, we calculate the difference in the average respiratory rate before $t$ and after $t$. Note that this measure assumes that the individual has been smoking for the entirety of the data up to the point of cessation. Fig.6 shows a plot of this for each day of the two individuals. The maximum change (the "respiratory rate baseline change peak") occurs a day after the actual day of cessation for subject A, with a change of 1.66 counts $/ \mathrm{min}$, and 51 days before for subject 2, with a change of 1.06 counts/min.

\section{Prediction of individuals who quit smoking}

If we can assume that the only effector that will cause the respiratory rate baseline to fall is the cessation of cigarettes, then the peak respiratory baseline change value (the height of the peak in Fig. 6) may in itself serve as a indicator of whether the individual has quit smoking. Fig. 7 shows the distribution of peak respiratory rate baseline change values for all individuals in our dataset. The mean is 0.22 counts/ min $(\sigma: 0.43)$, which is significantly lower than our two individuals who quit smoking. In fact, setting a threshold at 0.65 counts $/ \mathrm{min}$ (i.e. $1 \sigma$ ) lets us cleanly separate individuals who quit smoking from the rest. Again, this result is only preliminary since we did not have enough to evaluate using independent training and test data.

\section{CONCLUSION AND FUTURE WORK}

In this study, we demonstrated that respiratory rate data estimated from wrist-based accelerometers can detect various physical conditions such as subjective inebriation, fever, and smoking cessation. We can predict days with high subjective inebriation far above chance level. We have been able to visualise days with fever, although the lack of data has stopped us from evaluating its predictive power. Similarly, we have shown that respiratory rate may allow us to identify individuals who quit smoking.

Many of the results in this study were based on a small number of unnormalised subjective self-reports, so the findings are not immediately generalizable. However, the results encourage us to believe that actigraphs may allow us to predict the three physical conditions we targeted in this study for a wider general population in the future.

The largest contribution of our work is in showing that actigraphs, which have previously only been considered as tools for behavioural recognition or measuring activity levels, have a potential to quantify more qualitative aspects of the human lifestyle. It is true that many of the matters covered in this paper can already be detected more accurately by other means; inebriation can be measured by blood alcohol level, fever can be detected by a thermometer, and smoking cessation could be hand logged. The importance of our findings is that we showed that all of these can be detected by a single, low cost, non-invasive sensor that is already widely distributed in consumer channels. We plan to conduct a more large scale, rigorous validation of the claims laid out in this paper, and consider consumer applications of our findings.

\section{REFERENCES}

1.Suzuki, K., Kuriyama, H., Yamashita, S., and Yano, K., "Life microscope: future of human, sensor and IT that 20 life tapestries tell." Hitachi Hyoron 89,12 (2007): 30-35.

2.R.J.Cole, D.F.Kripke, W.Gruen, D.J.Mullaney, and J.C.Gillin, "Automatic Sleep/Wake Identification From Wrist Activity," Sleep, 15,5 (1992), 461-469.

3.Ravi, N., Dandekar, N., Mysore, P., and Littman, M. L., "Activity recognition from accelerometer data." AAAI, 5 (2005) 1541-1546.

4.Kawamoto, K., Hiroyuki, K., and Seiki T. "Actigraphic Detection of REM Sleep Based on Respiratory Rate Estimation." Journal of Medical and Bioengineering, 2, 1 (2013).

5.Littner, M., et al., "Practice parameters for using polysomnography to evaluate insomnia: An update.," Sleep, 26,6 (2003), 754-760.

6.Fitbit website, http://www.fitbit.com

7.Jawbone Up website, https://jawbone.com/up

8.Nike+ Fuelband SE website, http://www.nike.com/us/ en_us/c/nikeplus-fuelband 